\title{
"Tamo Junto": Intervenção breve em cumprimento de medida socioeducativa por uso de drogas
}

\author{
Geovani Garcia Zeferino \\ Anna Clara Santos da Silva \\ Andréa Cabral Rios
}

\section{RESUMO}

Segundo a legislação vigente, a pessoa que adquirir, portar ou guardar drogas ilícitas, pode ter o comportamento classificado como uso próprio ou tráfico, dependendo da situação e antecedentes. Em parceria com o Juizado Especial, o curso de Psicologia de uma cidade de Minas Gerais implantou uma modalidade para execução de medidas socioeducativas baseada na metodologia da Intervenção Breve para uso de drogas, aplicada ao contexto penal. A intervenção objetivou prevenir problemas relacionados ao uso de drogas e novas reincidências decorrentes do consumo. 0 presente estudo apresenta dados preliminares de três semestres dessa intervenção, a saber: aderência ao programa, perfil das pessoas, drogas mais utilizadas e considerações acerca da ocorrência. Foram atendidas 53 pessoas, predominantemente homens $(96,2 \%)$ entre 18 e 44 anos de idade (média $=24,8)$. Dessas, 34 concluíram, sete não concluíram e 12 não aderiram aos atendimentos. Os instrumentos utilizados foram AUDIT e ASSIST. As drogas mais consumidas foram: maconha (82,9\%), cocaína $(12,2 \%)$ e crack $(4,9 \%)$. 0 estudo aponta a importância do desenvolvimento de novas práticas conciliatórias entre o sistema penal e acadêmico técnico para evitar agravamentos de problemas relacionados às drogas, diminuir a prática punitiva e aumentar alternativas preventivas de intervenção.

Palavras-chave: intervenção breve; medida socioeducativa; uso problemático de drogas ilícitas.

\section{ABSTRACT}

\section{"Tamo Junto": Brief intervention in educative measure by use of drugs}

According to the current legislation, people who purchase, carry or keep illicit drugs may be classified as drug dealers or users, depending on the situation and criminal record. In partnership with the Special Court, the Psychology course of a city in the state of Minas Gerais developed an alternative for complying with socio-educational measures due to drug abuse, based on the methodology of Brief Intervention for such issue, applied to the criminal context. The intervention aimed to prevent drug use and recidivism problems. This study presents preliminary data of three semesters of implementation of this intervention, namely: program adherence, attended persons' profile, most used drugs and considerations of the individuals about the occurrence. Fifty-three people were seen, predominantly men $(96.2 \%)$ between 18 and 44 years old (average $=24.8$ ). Thirty-four persons concluded the consultations, while seven did not and the other 12 did not join the program. The tests used were AUDIT and ASSIST. The most consumed drugs were marijuana $(82.9 \%)$, cocaine $(12.2 \%)$ and crack $(4.9 \%)$. The study shows the importance of developing new conciliatory practices between the penal and the technical academic system to avoid the aggravation of drug problems, reduce punitive practices and increase preventive intervention alternatives.

Keywords: brief intervention; socio-educational measure; problematic use of illicit drugs.

\section{Sobre os Autores}

G.G.Z.

orcid.org/0000-0002-8245-668x Centro Universitário de Lavras,

Lavras, MG

geogarciapsico@gmail.com

A.C.S.S.

orcid.org/0000-0002-0831-6793 Centro Universitário de Lavras, Lavras, MG annaclarapsico@hotmail.com

A.C.R. orcid.org/0000-0001-6289-8942 Centro Universitário de Lavras, Lavras, MG

deia_rios@yahoo.com.br

\section{Direitos Autorais}

Este é um artigo de acesso aberto e pode ser reproduzido livremente, distribuído,

transmitido ou modificado, por qualquer pessoa desde que usado sem fins comerciais. $O$ trabalho é disponibilizado sob a licença Creative Commons CCBY-NC. 
O uso de drogas é algo frequente na sociedade e seu consumo pode ser feito por várias razões, que abrangem até mesmo rituais religiosos e procedimentos medicinais. A utilização também é feita pelo prazer que a substância gera e isso se constitui em um problema na saúde pública mundial (Marangoni \& Oliveira, 2013). Esse uso é capaz de modificar aspectos físicos, psicológicos e sociais na vida do sujeito, e pode acarretar dependência aos usuários e prejuízos a seus familiares (Medeiros, Maciel, Sousa, Tenório-Souza, \& Dias, 2013; Ronzani, 2013).

Além desses prejuízos, destacam-se os problemas de saúde pública, os quais necessitam de condutas conscientizadoras com populações vulneráveis (Schenker \& Minayo, 2003). Tais condutas buscam incentivar formas de convivência social mais saudáveis a essas pessoas, de forma a evitar o efeito de políticas de isolamento, punição e repressão (Granja, Gomes, Medrado, \& Nogueira, 2015). Partindo desse pressuposto, Boiteux e Ribeiro (2010) afirmam que o uso de drogas se tornou uma adversidade de cunho social e de saúde, no qual necessita de promoção de políticas públicas mais abrangentes e menos repressoras. Essas políticas públicas partiriam do princípio de não tratar o usuário como criminoso ou doente (Nascimento, 2006).

Essas repressões são caracterizadas como crime quando há produtividade sem autorização e tráfico ilícito de substâncias (Institui o Sistema Nacional de Políticas sobre Drogas SISNAD, 2006). Baseado na lei $11.343 / 2006$, as drogas são classificadas em duas categorias, a saber: ilícitas (quando a produção, comercialização e uso não são permitidos por lei), e lícitas (quando estão disponíveis e podem ser vendidas segundo a constituição brasileira, como o tabaco e o álcool (Institui o Sistema Nacional de Políticas sobre Drogas - SISNAD, 2006). No Brasil, historicamente, a legislação age com medidas de políticas proibicionista e punitivas. A antiga lei $6.368 / 1976$, que vigorou por 30 anos, propunha medidas que buscavam a proibição do uso e comercialização de drogas consideradas ilícitas, aumentando os estereótipos dos usuários que eram chamados de "drogados", "vagabundos", contribuindo para a sua marginalização (Santos, Acioli Neto, Galindo, \& Souza, 2015). Porém, com a implantação da Lei, $11.343 / 2006$ o usuário passou a ser tratado diferente do traficante em relação à pena.

0 artigo $1^{\circ}$ da Lei $n^{\circ} 11.343 / 2006$, propõe ações preventivas ao uso considerado inadequado de drogas, atenção e reinserção social dos usuários e dependentes. 0 artigo 28 desta mesma lei considera usuário quem adquire, porta, transporta ou traz consigo, para uso próprio, drogas sem autorização ou em desacordo com determinação legal ou regulamentar. Caso considerado traficante, o infrator é enquadrado no artigo 33 da mesma lei, que caracteriza como tráfico de drogas, a venda, oferta, estoque, entrega ou fornecimento, mesmo que gratuito. 0 que difere, inicialmente, é a avaliação da autoridade competente pela ocorrência, o histórico da pessoa e a situação em que a droga se encontra e, posteriormente, o juiz avaliará a quantidade de droga apreendida, o local e situação do evento, o contexto social e histórico pessoal (Institui o Sistema Nacional de Políticas sobre Drogas - SISNAD, 2006).

O sujeito abordado e enquadrado no artigo 33 dessa lei é investigado e avaliado posteriormente por autoridade qualificada, ou seja, por Juiz de direito, para julgar sua situação com a justiça, com pena de reclusão de 5 (cinco) a 15 (quinze) anos. Já em situação de uso, artigo 28, pode-se designar pena de advertência, prestação de serviço à comunidade ou medida educativa. Quanto às penas de cumprimento de medida educativa, o inciso III do artigo 28, visa oferecer atendimento e orientação para demonstrar agravantes jurídicos, sociais ou pessoais, no intuito de reduzir danos gerados pelo uso da droga (Institui o Sistema Nacional de Políticas sobre Drogas - SISNAD, 2006).

Para Oliveira e Jesus (2014), a intenção dessa Lei representa a busca por diferenciação entre usuário e traficante através da descriminalização do porte de drogas para uso próprio, presente nos artigos 28 (uso de drogas) e 33 (tráfico de drogas), ou seja, demonstra uma preocupação com a recuperação dos usuários que necessitam de apoio ao invés de ser penalizados pela justiça com privação de liberdade, e ao mesmo tempo salienta penas mais severas para os traficantes.

Souza (2006) aponta que leis são um respaldo para que as políticas públicas aconteçam e se tornem uma ferramenta relevante na estrutura coletiva para que exerça papel importante na evolução da sociedade. Dentro dessa perspectiva de evolução cria-se a possibilidade de extinguir a repressão pelo uso de drogas. Para tanto, têm-se políticas públicas focadas na Redução de Danos, ou seja, seguindo o pensamento de que o Estado precisa admitir que o uso de drogas não irá cessar e a partir daí, investir em políticas que visam diminuir as influências danosas que podem se originar através do uso de substâncias psicoativas, que refletem tanto no indivíduo, quanto na sociedade (Souza, 2015).

Dentro dessa perspectiva as estratégias que visam diminuir os efeitos danosos do consumo abusivo de substâncias ou prevenir seu agravamento, as técnicas de curta duração são relevantes no tratamento do uso abusivo de álcool e outras drogas, tanto no cenário nacional (Bandinelli, Gonçalves, \& Fonseca, 2013; Carvalho, 2015; Novaes, 2014; Souza, Luis, \& Corradi-Webster, 2013), quanto internacional (Mdege \& Watson, 2013; Patton et al., 2014; Schonfeld et al., 2015).

Dentre essas técnicas de curta duração, a Intervenção 


\section{W'INTERACÃO EM ET. PSICOLOGIA}

Breve (IB) é destacada por ser uma modalidade interventiva que busca motivar a mudança de comportamentos ligados ao uso de drogas e auxilia na identificação de situações e motivos que possam gerar prejuízos recorrentes do uso de substâncias psicoativas (Gigliotti, 2010; Marques \& Furtado, 2004; Silva \& Miguel, 2011; Souza \& Ronzani, 2012). Gigliotti (2010) ainda acrescenta que essa técnica possui baixo custo e simplicidade em sua aplicação.

Ferreira, Albertoni, Silva e Sartes (2016) também destacam a contribuição da IB no contexto de álcool e outras drogas, demonstrando, no âmbito laboral no consumo de álcool, que os participantes da IB diminuíram consideravelmente o uso de álcool, em relação a outro grupo que apenas recebeu panfletos e feedback.

A IB é uma modalidade de intervenção considerada eficaz para prevenir problemas decorrentes do uso abusivo de álcool e outras drogas, sendo utilizada em vários contextos de atenção em saúde. Nesse tipo de intervenção, não se busca realizar diagnóstico de dependência de álcool e outras drogas, mas apontar problemas a serem avaliados com cautela, como o uso indevido de drogas, que posteriormente, são tratados de maneira direta (Silva \& Miguel, 2011). A utilização de IB é mais indicada nos casos de usuários de risco ou uso nocivo, pois nos casos de dependência a medida não possui tanta eficácia (Marques \& Furtado, 2004).

O foco da IB é a mudança de comportamento do usuário, sem confronto ou julgamento. Ela busca compreender e orientar a pessoa atendida, no intuito de diminuir o consumo (Silva \& Tucci, 2015), e, ao mesmo tempo, estimular a reflexão acerca dos riscos que o uso abusivo de drogas pode causar. Diante disso, criam-se de maneira conjunta (usuário e terapeuta), metas individuais para continuar reduzindo ou, até mesmo, cessar o uso da substância (Babor, Higgins-Biddle, Saunders, \& Monteiro, 2001; Marques \& Furtado, 2004).

Diante do exposto, o presente trabalho apresenta a aplicação do modelo de IB no atendimento a adultos cumprindo medida socioeducativa por uso ilegal de drogas ou outros problemas relacionados. Para tanto, são apresentados dados descritivos preliminares, referentes às atividades promovidas em três semestres letivos por estagiários de Psicologia no atendimento a tais pessoas.

\section{MÉTODO}

O projeto "TAMO JUNTO" - Intervenção Breve a pessoas com problemas decorrentes do uso de substâncias psicoativas, constitui-se em uma atividade de extensão acadêmica realizada por graduandos de Psicologia de um Centro Universitário em Minas Gerais, em parceria com o Juizado Especial da mesma cidade. Iniciou suas ações no ano de 2015 junto às pessoas abordadas por porte de drogas ilícitas e penalizadas criminalmente como usuárias. 0 programa busca constituir-se em uma modalidade alternativa de intervenção ao uso indevido de substâncias ilícitas, com atuação focada nos sujeitos penalizados para auxiliar na avaliação e reflexão acerca de comportamentos de risco e, dessa forma, tentar diminuir/cessar as reincidências em delitos referentes ao consumo de drogas ilícitas, através de ofertas de medidas socioeducativas de conscientização e prevenção ao uso problemático de drogas.

Inicialmente, foi constituída uma equipe de trabalho composta por treze alunos e a professora coordenadora. Os alunos participaram de um grupo de estudos sobre álcool e outras drogas de modo a fundamentar conhecimentos sobre tipos de substâncias, classificações internacionais, critérios diagnósticos, instrumentos de avaliação e perspectivas de intervenção, prevenção e redução de danos. Posteriormente, os alunos participaram de audiências relacionadas a uso e/ou porte de drogas, visando acompanhar como se estabelecia o processo de indicação de penas alternativas. A partir de observações realizadas previamente, o projeto e a proposta de intervenção foram formatados, bem como os instrumentos de atendimento, avaliação e intervenção.

Depois de apresentada a proposta de atuação, ela foi aprovada pelo Juiz e pelo Promotor do Juizado, assim como pela Coordenadoria de Extensão da Instituição e o comitê de ética em pesquisa do Centro Universitário de Lavras (CAAE n.59502016.0.0000.5116). As atividades foram realizadas no período de agosto de 2015 a dezembro de 2016. Ao comparecer à audiência, os usuários sob pena do inciso III do artigo 28 , referente à Lei $n^{\circ} 11.343 / 06$ foram informados previamente sobre a medida socioeducativa e lhes era dada a opção de cumprir serviços comunitários, pagar cestas básicas ou comparecer a três sessões de Intervenção Breve, oferecidas pelos estagiários. Aqueles que optaram pelo comparecimento às sessões de Intervenção Breve agendavam no momento da audiência as sessões realizadas, preferencialmente, em três semanas consecutivas.

\section{PARTICIPANTES}

A amostra foi composta por 53 pessoas (de ambos os sexos), com idade entre 18 e 44 anos, que compareceram à audiência de instrução e julgamento e optaram pela medida socioeducativa ao invés de prestação de serviço à comunidade ou multa. A análise dos processos permitiu observar que 18 dessas pessoas já haviam enfrentado problemas com a justiça anteriormente devido ao uso de drogas.

A coleta de dados ocorreu em uma sala do Juizado Especial, destinada aos atendimentos. 


\section{W'INTERACÃO EM ET PSICOLOGIA}

\section{INSTRUMENTOS}

A intervenção compunha-se de três sessões, nas quais eram utilizados formulários elaborados previamente pela coordenadora e equipe acadêmica, no intuito de padronizar aspectos essenciais à metodologia proposta, como as questões inicialmente abordadas e instrumentos utilizados. Partindo desse formulário, os instrumentos adotados foram o Alcohol Use Disorder Identification Test (AUDIT) e Alcohol, Smoking and Substance Involvement Screening Test (ASSIST).

O AUDIT tem a versão original desenvolvida pela Organização Mundial de Saúde (OMS) (Babor, Higgins-Biddle, Saunders, \& Monteiro, 2001) e validado no Brasil por Méndez, Lima, Olinto e Farrel (1999). O teste contém dez itens que avaliam, nos últimos doze meses, a frequência do consumo de álcool, indício de dependência da substância, perda de memória, ação que possa gerar ferimento em si próprio ou em outra(s) pessoa(s) e efeitos que possam prejudicar o usuário devido ao consumo excessivo. Esse instrumento pode ser autoaplicável ou utilizado em forma de entrevista (Micheli, Formigoni, Ronzani, \& Carneiro, 2017).

O ASSIST foi desenvolvido pelo WHO ASSIST Working Group (2002) e validado no Brasil por Henrique, Michelli, Lacerda, Lacerda e Formigoni (2004). 0 teste possui oito questões e avalia 0 uso de drogas na vida e nos três últimos meses, os riscos atuais e futuros em decorrência do uso, evidência de dependência (questões de um a sete) e utilização de drogas injetáveis (questão oito). Além disso, é um teste padronizado, de rápida aplicação, com questionamentos de várias substâncias e fácil interpretação (Henrique et al., 2004).

\section{PROCEDIMENTOS}

No primeiro contato com o usuário era feito o acolhimento empático do cliente e enquadre das atividades. 0 atendido era inicialmente informado acerca da estrutura proposta (três sessões) e objetivos da atividade e das sessões. Nesse momento, a pessoa era informada acerca dos princípios éticos dos atendimentos (uso de dados produzidos para fins de estudos, anonimato, sigilo das informações, riscos de ordem física e/ou moral a ele e benefícios). Por fim, caso consentisse em participar das atividades propostas era assinado o Termo de Consentimento Livre e Esclarecido com as datas e horários dos dois próximos atendimentos, bem como autorização para utilização de dados em futuras pesquisas.

0 atendimento inicial, em seguida, consistia no levantamento de informações na perspectiva do atendido sobre:

- Situação conjugal, escolaridade, idade, ocupação atual, renda familiar, plano de saúde;
- O histórico da ocorrência policial e a que causas a atribuía;

- Presença de outras ocorrências e/ou problemas policiais ou judiciais;

- Utilização de medicamentos (psiquiátricos ou não)

- Avaliação subjetiva do seu uso de substâncias e como os familiares avaliam esse uso;

- Presença de problemas físicos, pessoais, sociais, de trabalho, afetivos ou familiares no passado que Ihe fossem significativos;Histórico de atendimento psicológico ou psicoterapêutico anterior;

- Opinião a respeito da atividade proposta a ele como medida socioeducativa na prevenção a problemas futuros decorrentes do consumo de drogas.

A segunda sessão era iniciada com uma discussão da sessão anterior, por parte do estagiário e da pessoa em atendimento e realizada a aplicação de inventários para avaliação de padrões de consumo de drogas. Primeiramente era usado o instrumento AUDIT e posteriormente o instrumento ASSIST. Por meio deles obtiveram-se informações sobre fatores de risco ao uso indevido de substâncias psicoativas, no intuito de promover uma reflexão sobre a relação do usuário com $\mathrm{a}(\mathrm{s})$ droga(s) de consumo e possíveis problemas decorrentes, caso houvesse. Com tal objetivo, eram feitos questionamentos:

- Se o atendido tinha alguma dúvida acerca dos efeitos da substância, meios de ajuda ou tratamentos para uso problemático de drogas;

- Se identificava alguma situação, dificuldade ou problema que estimulava seu consumo de substâncias e;

- Já tinha feito algum tipo de tratamento ou buscou algum tipo de ajuda para uso de drogas e, se sim, como tinha sido.

$\mathrm{Na}$ terceira sessão iniciava-se com a devolutiva dos testes e solicitava-se uma autoavaliação dos resultados dos instrumentos utilizados, bem como apontar seu padrão de consumo de álcool e outras drogas e as reflexões suscitadas a partir dos resultados. Por fim, era feito um fechamento com a pessoa atendida, solicitando-se um feedback acerca das atividades realizadas nos três encontros e questionando a pessoa atendida sobre sua percepção do que podia estimular o uso indevido de drogas. Por fim, o participante era indagado sobre seu interesse em implantar medidas de prevenção da evolução de seus padrões de consumo ou redução de danos futuros, discutindo, se necessário, opções para tanto, além de fornecer informações acerca dos serviços de atenção e, caso fosse de seu interesse, o encaminhamento à Clínica-escola de Psicologia e serviços públicos e comunitários da cidade. 


\section{W'INTERACÃO EM ET PSICOLOGIA}

\section{RESULTADOS}

Das 53 pessoas atendidas, 34 pessoas $(64,1 \%)$ concluíram o atendimento, 12 (22,6\%) não compareceram a nenhum atendimento e sete pessoas $(13,2 \%)$ iniciaram e não a concluíram. Dessas 34 pessoas que concluíram os atendimentos havia apenas dois casos $(3,8 \%)$ do sexo feminino. Elas cumpriram as três sessões sem a necessidade de remarcar algum dos atendimentos.

Em relação ao tipo de droga ilícita mais utilizada, a maconha foi a mais identificada, em $82,9 \%$ nos casos. A cocaína foi a segunda, com $12,2 \%$ e o crack a terceira droga ilícita mais usada, com $4,9 \%$ dos casos. Acerca das drogas lícitas o álcool foi o mais usado pela população desse estudo. Aproximadamente $87,8 \%$ dos participantes relataram que consumem algum tipo de bebida alcoólica com frequência e 41,5\% da amostra relataram usar tabaco. Por fim, 40 participantes $(75,5 \%)$ relataram que fazem uso de duas ou mais drogas com frequência.

\begin{tabular}{|c|c|}
\hline Droga usada & Número de usuários \\
\hline Maconha & 34 \\
\hline Cocaína & 5 \\
\hline Crack & 2 \\
\hline Extase & 0 \\
\hline Alucinógenos & 2 \\
\hline Opióides ou opiáceos & 0 \\
\hline Álcool & 36 \\
\hline Tabaco & 7 \\
\hline Anfetamina & 2 \\
\hline Hipnóticos ou sedativos & 1 \\
\hline
\end{tabular}

Em relação às considerações dos participantes acerca da ocorrência, 38 pessoas (71,7\%) alegaram terem sido abordadas pela polícia por "uma bobeira" ou "descuido", 5 pessoas $(9,4 \%)$ relataram que deram azar em estar no lugar errado e na hora errada e 10 pessoas $(18,9 \%)$ disseram que não tinham culpa ou foram injustiçados. Das 53 pessoas atendidas, $22(41,5 \%)$ disseram no primeiro atendimento que aquela seria a primeira vez que estavam usando algum tipo de droga ilícita. Porém ao analisar os processos desses participantes, foi possível verificar que 18 deles (34\%) já tinham outra(s) ocorrência(s) envolvendo algum tipo de droga.

\section{DISCUSSÃO}

Granja et al. (2015) afirmam que os homens tendem a usar mais drogas, tanto por fatores culturais, quanto pela aceitação social. Os autores ainda destacam que os serviços de políticas de saúde sobre drogas no Brasil normalmente são criados para atender a demanda masculina, na maior parte dos casos, e com o surgimento da demanda feminina os mesmos serviços sofrem uma adequação para serem oferecidos. Tais informações corroboram com os resultados obtidos nas análises dos prontuários em relação ao sexo dos atendidos, uma vez que a maioria dos atendidos era do sexo masculino.

O perfil de pessoas atendidas é similar ao observado no II Levantamento Nacional de Álcool e Drogas realizado pelo Instituto Nacional de Ciência e Tecnologia para Políticas Públicas do Álcool e Outras Drogas (INPAD), a maconha é a droga ilícita de maior consumo na população em geral (Laranjeira, 2014; vide também Piccoli e Rodrigues, 2016).

No que tange às contradições das informações sobre as ocorrências envolvendo substâncias psicoativas, trazidas pelos atendidos e verificadas no prontuário, pode-se considerar que segundo Knauth, Pilecco, Leal, Seffner e Teixeira (2012), o dia-a-dia de usuários de drogas é regulado pelo sentimento de medo e estigma, englobando todas as classes e categorias no meio social. Dessa forma, constatou-se que a mentira era acompanhada de medo e vergonha gerados pelo estigma causado em decorrência do uso de drogas. A mentira não era confrontada pelos estagiários, haja vista que esse não é o intuito da IB, porém utilizavam-se algumas alternativas para causar reflexão no usuário. Buscava-se trabalhar com situações do tipo: "e se você tivesse outra(s) ocorrência(s), como seria?", "Como agiria?", etc.

A IB foi utilizada nesta ocasião juntamente com a redução de danos, como sugere Marques e Furtado (2004) ao destacarem que é necessário, primeiramente, identificar situaçõesproblema e, posteriormente, iniciar o encorajamento para superá-las. Foram oferecidos em curto prazo informações sobre as substâncias e seus efeitos e malefícios, riscos ligados à saúde e eventuais problemas com a justiça, para que o usuário reflita sobre sua atual situação e crie alternativas para mudar comportamentos indesejados. Em longo prazo, foram sugeridas tarefas graduais para estabelecer compromisso com o tratamento e proposto encaminhamento para psicoterapia.

Acerca da adesão do programa, pode-se verificar um aproveitamento de $64,1 \%$. Isso se consolidou, principalmente, 
pela proposta de redução de danos no sentido de a pessoa atendida participar ativamente do atendimento, aderindo ao compromisso de se cuidar, ter a opção de realizar suas escoIhas e criar suas próprias metas para evitar novos prejuízos. Ao mesmo tempo, visou-se diminuir os agrupamentos identitários, que se referem a usuários abusivos de substâncias psicoativas como drogados, doentes e criminosos (Santos \& Ferla, 2017). Dessa forma a IB se caracterizou nesses atendimentos como uma medida alternativa, no intuito de fornecer atendimentos empáticos aos usuários que cumpriram a proposta. Aos que não foram contemplados com a medida, não foi possível possibilitar alguma mudança de hábito em relação às substâncias psicoativas.

Alguns estudos utilizaram a IB como metodologia em uso de drogas, porém em outras populações, a saber, adolescentes (Patton et al., 2014); estudantes universitários (Silva \& Tucci, 2015); e idosos (Schonfeld et al., 2015); e em outros contextos, como aposentadoria (França, Murta, Negreiros, Pedralho, \& Carvalhedo, 2013); ambiente de trabalho (Ferreira et al., 2016); e atenção primária de saúde (Carvalho, 2015; Laport, Costa, Mota, \& Ronzani, 2016). Contudo, há uma lacuna de estudos voltados para a população específica focada no presente artigo, justificando a necessidade de novos estudos e a replicação da presente metodologia.

\section{CONSIDERAÇÕES FINAIS}

Um dos desafios da Lei 11.343/06 é propor uma diferenciação objetiva entre o uso e tráfico de drogas, ou seja, distinguir a pessoa nos artigos 28 e 33 . A partir da demanda apresentada pelo Juiz, o projeto buscou, primeiramente, trabalhar com o intuito de não criminalizar ou patologizar os usuários. 0 foco dos atendimentos não foi atender o criminoso, usuário ou doente, mas questionar uma perspectiva moralista e/ou proibicionista em relação ao uso de drogas e aderir a uma visão mais preventiva, que atue com propostas de redução de danos ou evolução dos padrões de consumo que podem gerar problemas futuros.

Nessa perspectiva, ao considerar o sujeito que faz o uso de substâncias, Nascimento (2006) aponta que, nesse contexto de políticas públicas voltadas para os problemas relacionados às drogas, existem duas visões norteadoras (a do usuário como um criminoso ou como o portador de uma doença). Por meio de uma visão crítica, o autor argumenta que as duas assumem uma postura punitiva. Diante dessas condições, o propósito do programa "Tamo Junto" foi atuar conjuntamente com o sujeito, abordando sua história, as questões que levaram o uso de drogas, mesmo que de maneira inicial. Desta forma, visou-se promover o início de uma reflexão que o ajudará a traçar os planos da intervenção, ou seja, trabalhar de maneira a não rotular o sujeito como um criminoso ou mesmo um doente.

Espera-se, portanto, que esse trabalho possa incentivar o desenvolvimento de novas práticas conciliatórias do sistema penal para abranger mais usuários de outras substâncias, e atender pessoas que apresentem problemas iniciais para que estes não se agravem ou se tornem dependência. Dessa forma, atua-se diretamente nessa população para o cumprimento desse tipo de medida configurada como alternativa preventiva a futuros problemas relacionados ao consumo de drogas e quebra de paradigmas e estereótipos, de forma não punitiva. Assim, é possível contribuir com a melhoria da saúde pública e auxiliar a comunidade em termos de tráfico de drogas, violência, roubos e outros aspectos relacionados às drogas.

\section{CONTRIBUIÇÃO DE CADA AUTOR}

G.G.Z e A.C.S.S contribuiram para a conceitualização, investigação, visualização e redação (rascunho) do artigo. G.G.Z, A.C.S.S e A.C.R. realizaram a redação (revisão e edição), e a supervisão ficou a encargo de A.C.R.

\section{DECLARAÇÃO DE CONFLITOS DE INTERESSE}

Os autores declaram que não há conflitos de interesse no presente artigo.

\section{REFERÊNCIAS}

Babor, T. F., Higgins-Biddle, J. C., Saunders, J. B., \& Monteiro, M. G. (2001). The alcohol use disorders identification test: Guidelines for use in. Primary Care (2 ed.). Genebra, Suíça: World Health Organization.

Bandinelli, L. B., Gonçalves, H. A., \& Fonseca, R. P. (2013). A entrevista motivacional e sua aplicabilidade em diferentes contextos: Uma revisão sistemática. Diaphora, 13(1), 2634.

Boiteux, L. \& Ribeiro, M. M. (2010). Justiça terapêutica: Redução de danos ou proibicionismo dissimulado? Em S. D. Seibel (Org.), Dependência de drogas (2 ed., pp. 26-37). São Paulo: Atheneu.

Carvalho, C. A. (2015). Avaliação da intervenção breve em tabagismo: Estudo piloto de um ensaio clínico randomizado (Dissertação de mestrado, Universidade Federal de Juiz de Fora - UFJF). Recuperado de https://repositorio.ufjf.br/jspui/bitstream/ufjf/376/1/clau diaaparecidadecarvalho.pdf. 
Ferreira, M. L., Albertoni, M. R., Silva, N. B., \& Sartes, L. M. A. (2016). Avaliação da efetividade da intervenção breve para a prevenção do uso de álcool no trabalho. Psicologia em Pesquisa, 10(1), 34-43.

http://dx.doi.org/10.24879/201600100010045.

França, C. L., Murta, S. G., Negreiros, J. L., Pedralho, M., \& Carvalhedo, R. (2013). Intervenção breve na preparação para aposentadoria. Revista Brasileira de Orientação Profissional, 14(1), 99-110.

Gigliotti, A. (Ed.). (2010). Diretrizes gerais para tratamento da dependência química (1 ed.). Rio de Janeiro: Rubio.

Granja, E., Gomes, R., Medrado, B., \& Nogueira, C. (2015). O (não) lugar do homem jovem nas políticas de saúde sobre drogas no Brasil: Aproximações genealógicas. Revista Ciência \& Saúde Coletiva, 20(11), 3447-3455. http://dx.doi.org/10.1590/1413812320152011.19972014.

Henrique, I. F. S., Michelli, D., Lacerda, R. B., Lacerda, L. A., \& Formigoni, M. L. O. S. (2004). Validação da versão brasileira do teste de triagem do envolvimento com álcool, cigarro e outras substâncias (ASSIST). Revista da Associação Médica Brasileira, 50(2), 199-206.

http://dx.doi.org/10.1590/S0104-42302004000200039.

Institui o Sistema Nacional de Políticas sobre Drogas SISNAD, Lei $n^{\circ}$ 11.343, Diário Oficial da União de 26 de agosto $\S 1$ (2006).

Knauth, D. R., Pilecco, F. B., Leal, A. F., Seffner, F., \& Teixeira, A. M. F. (2012). Manter-se acordado: A vulnerabilidade dos caminhoneiros no Rio Grande do Sul. Revista de Saúde Pública, 46(5), 886-893.

http://dx.doi.org/10.1590/S0034-89102012000500016.

Laport, T. J., Costa, P. H. A., Mota, D. C. B., \& Ronzani, T. M. (2016). Percepções e práticas dos profissionais da atenção primária à saúde na abordagem sobre drogas. Psicologia: Teoria e Pesquisa, 32(1). http://dx.doi.org/10.1590/0102-37722016012055143150.

Laranjeira, R. (Org.). (2014). II Levantamento Nacional de Álcool e Drogas (LENAD) - 2012. São Paulo: Instituto Nacional de Políticas Públicas do Álcool e Outras Drogas (INPAD). Recuperado de http://inpad.org.br/wpcontent/uploads/2014/03/Lenad-II-Relat\%C3\%B3rio.pdf.

Marangoni, S. R., \& Oliveira, M. L. F. (2013). Fatores desencadeantes do uso de drogas de abuso em mulheres. Texto \& Contexto Enfermagem, 22(3), 662-670. http://dx.doi.org/10.1590/S0104-07072013000300012.

Marques, A. C. P. R., \& Furtado, E. F. (2004). Intervenções breves para problemas relacionados ao álcool. Revista Brasileira de Psiquiatria, 26(1), 28-32.

http://dx.doi.org/10.1590/S1516-44462004000500008.
Mdege, N. D., \& Watson, J. (2013). Predictors of study setting (primary care vs. hospital setting) among studies of the effectiveness of brief interventions among heavy alcohol users: A systematic review. Drug and Alcohol Review, 32(4), 368-380. http://dx.doi.org/10.1111/dar.12036.

Medeiros, K. T., Maciel, S. C., Sousa, P. F., Tenório-Souza, F. M., \& Dias, C. C. V. (2013). Representações sociais do uso e abuso de drogas entre familiares de usuários. Psicologia em estudo, 18(2), 269-279.

http://dx.doi.org/10.1590/S1413-73722013000200008.

Méndez, E. B., Lima, M. S., Olinto, M. T. A., \& Farrel, M. (1999). Uma versão brasileira do AUDIT-Alcohol Use Disorders Identification Test (Dissertação de mestrado, Faculdade de Medicina, Universidade Federal de Pelotas). Recuperado de http://www.epidemioufpel.org.br/uploads/teses/Brod\%20Mendez\%201999\%20 Dissert.pdf.

Micheli, D., Formigoni, M. L. O. S., Ronzani, T. M., \& Carneiro, A. P. L. (2017). Uso, abuso ou dependência? Como fazer triagem usando instrumentos padronizados. Em Secretaria Nacional de Políticas sobre Drogas - SENAD (Org.), Detecção do uso e diagnóstico da dependência de substâncias psicoativas (11a/12a ed., pp. 25-44). São Paulo: UNIFESP.

Nascimento, A. B. (2006). Uma visão crítica das políticas de descriminalização e de patologização do usuário de drogas. Psicologia em Estudo, 11(1), 185-190. http://dx.doi.org/10.1590/S1413-73722006000100021.

Novaes, P. S. (2014). 0 tratamento da dependência química e o ordenamento jurídico brasileiro. Revista Latinoamericana de Psicopatologia Fundamental, 17(2), 342-356. http://dx.doi.org/10.1590/1984-0381v17n2a13.

Oliveira, A. A. G., \& Jesus, M. M. G. (2014). A flexibilização da Igislação penal brasileira frente aos usuários de entorpecentes. Revista Transgressões Ciências Criminais em Debate, 2(1), 124-138.

Patton, R., Deluca, P., Kaner, E., Newbury-Birch, D., Phillips, T., \& Drummond, C. (2014). Alcohol screening and brief intervention for adolescents: The how, what and where of reducing alcohol consumption and related harm among young people. Alcohol and Alcoholism, 49(2), 207-212. http://dx.doi.org/10.1093/alcalc/agt165.

Piccoli, T. T., \& Rodrigues, A. D. (2016). Uso de cocaína e maconha no setor metalúrgico em Caxias do Sul, RS, Brasil: Uma revisão da literatura. Ciência em Movimento, 18(36), 9-16.

http://dx.doi.org/10.15602/19839480/cmbs.v18n36p916. 


\section{站 NTERACÃOEM ET. PSICOLOGIA}

Ronzani, T. M. (Org.). (2013). Ações integradas sobre drogas: Prevenção, abordagens e políticas públicas (1a ed.). Juiz de Fora: UFJF.

Santos, F. F. D., \& Ferla, A. A. (2017). Saúde mental e atenção básica no cuidado aos usuários de álcool e outras drogas. Interface-Comunicação, Saúde, Educação, Publicado adiantado em forma online. http://dx.doi.org/10.1590/1807-57622016.0270.

Santos, M. F. S., Acioli Neto, M. L., Galindo, F. S., \& Souza, L. B. (2015). A ambivalência no campo das drogas: Uma análise das representações de álcool e maconha. Revista de Administração Educacional, 1(2), 125-145.

Schenker, M., \& Minayo, M. C. S. (2003). A implicação da família no uso abusivo de drogas: Uma revisão crítica. Ciência \& Saúde Coletiva, 8(1), 299-306. http://dx.doi.org/10.1590/S1413-81232003000100022.

Schonfeld, L., Hazlett, R. W., Hedgecock, D. K., Duchene, D. M., Burns, L. V., \& Gum, A. M. (2015). Screening, brief intervention, and referral to treatment for older adults with substance misuse. American journal of public health, 105(1), 205-211. http://dx.doi.org/10.2105/AJPH.2013.301859.

Silva, C. J., \& Miguel, A. Q. C. (2011). Intervenção breve. Em A. Diehl, D. C. Cordeiro, \& R. Laranjeira (Ed.), Dependência química: prevenção, tratamento e políticas públicas (1 ed., pp. 243-251). Porto Alegre: Artmed.

Silva, E. C., \& Tucci, A. M. (2015). Intervenção breve para redução do consumo de álcool e suas consequências em estudantes universitários brasileiros. Psicologia: Reflexão e Crítica, 28(4), 728-736. http://dx.doi.org/10.1590/1678-7153.201528410.
Souza, A. L. (2015). Uso de drogas e acesso à saúde pública: Análise a partir das políticas públicas de redução de danos para usuários e dependentes (Trabalho de conclusão de curso, Universidade do Extremo Sul Catarinense - UNESC). Recuperado de http://200.18.15.27/bitstream/1/3748/1/ANNY\%20LIMA\% 20DE\%20SOUZA.pdf.

Souza, C. (2006). Políticas públicas: Uma revisão da literatura. Sociologias, 8(16), 20-45. http://dx.doi.org/10.1590/S1517-45222006000200003.

Souza, I. C. W., \& Ronzani, T. M. (2012). Álcool e drogas na atenção primária: Avaliando estratégias de capacitação. Psicologia em Estudo, 17(2), 237-246. http://dx.doi.org/10.1590/S1413-73722012000200007.

Souza, J., Luis, M. A. V., \& Corradi-Webster, C. M. (2013). Brief interventions and tools for nursing care: Descriptive and exploratory study. Online Brazilian Journal of Nursing, 12(1), 21-32. http://dx.doi.org/10.5935/1676-4285.20133504.

WHO ASSIST Working Group (2002). The alcohol, smoking and substance involvement screening test (ASSIST): Development, reliability and feasibility. Addiction, 97(9), 1183-1194. http://dx.doi.org/10.1046/j.1360-0443.2002.00185.x.

Artigo submetido em: 19/04/17 Primeira decisão editorial em: 08/06/17 Aceito em: 25/07/17 